\title{
Analysis of kinematic motion deviations of machining centers based on geometric tolerances
}

\author{
Atsushi TAKAHASHI*, Arata YOSHIDA*, Wiroj THASANA* \\ Nobuhiro SUGIMURA*, Koji IWAMURA* and Yoshitaka TANIMIZU* \\ * Graduate School of Engineering, Osaka Prefecture University \\ 1-1 Gakuen-cho, Naka-ku, Sakai, Osaka 599-8531, Japan \\ E-mail: sugimura@me.osakafu-u.ac.jp
}

Received 24 March 2014

\begin{abstract}
The objectives of the present research are to investigate relationships between kinematic motion deviations of machine tools and geometric tolerances of their components, to propose mathematical models representing kinematic motion deviations of five-axis machining centers, and to apply the models to analysis of the kinematic motion deviations of five-axis machining centers. A set of models is proposed to represent kinematic motion deviations of both the linear tables and the rotary tables, based on the geometric tolerances of the guide-ways connecting the components. By combing the models, three models of the five-axis machining centers are developed and applied to the analysis of the standard deviations of the shape generation motions of the tools and the workpieces. The proposed models provides us with a systematic method to analyze and to estimate the kinematic motion deviations of the five-axis machining centers, based on the geometric tolerances of the guide-ways connecting the components.
\end{abstract}

Key words : Five-axis machining centers, Shape generation motions, Geometric tolerances, Kinematic motion deviations

\section{Introduction}

Machining accuracy is one of the most important characteristics of the $\mathrm{CNC}$ machine tools for generating the products with the high accuracy and the complicated geometries. Some researches have been carried out to analyze the machining accuracy of the machine tools based on the shape generation motions between the tools and the workpieces (Sugimura, et al., 1981; Reshetov and Portman, 1988; Sakamoto and Inasaki, 1994; Sugimura, et. al., 1998). However, the kinematic motion deviations of the machine tools are deeply influenced by the geometric tolerances of the components, such as guide-ways and bearings. Therefore, it is required to clarify the relationships between the kinematic motion deviations of the machine tools and the geometric tolrenaces of the components, from the viewpoints of the design and the manufacturing of the machine tools and their components.

Many researches have also been carried out to deal with the dimensional tolerances and the geometric tolerances, aimed at realizing systematic analysis and design methodologies for the three dimensional machine products (Roy, et al., 1991; Voelcker, 1993; Nigam and Turner, 1995; Ngoi, et al., 2000). The relationships between the motion deviations and the geometric tolerances of the components have not yet been clarified.

The objectives of the present research are to establish mathematical models representing the kinematic motion deviations of the machine tools, on the basis of the geometric tolerances of the components, and to apply the models to theoretical analysis of the kinematic motion deviations of the machine tools. In the previous papers (Satonaka, et al., 2007; Satonaka, et al., 2008), a model has been proposed to represent the kinematic motion deviations of the linear tables, and the proposed model has been applied to the analysis of the standard deviations of the shape generation motions of the three-axis machining centers. The models of the tow-axis rotary tables have also been proposed, and 
applied to the estimation of the standard deviations of the rotary motions (Sugimura, et al., 2012). The issues discussed in the paper are summarized in the followings.

(1) Modelling of kinematic motion deviations for rotary tables and linear tables,

(2) Modelling of kinematic motion deviations for three types of five-axis machining centers, and

(3) Applications of proposed models to analysis of kinematic motion deviations of five-axis machining centers.

The objectives of the present paper is to establish mathematical models representing the kinematic motion deviations of the 5-axis machining centers, on the basis of the geometric tolerances of the components, and to apply the models to the standard deviation analysis of the kinematic motions of the 5-axis machining centers. The kinematic motion deviations are estimated based on the models in the various conditions of the geometric tolerances of the components and the various table positions, to investigate the influence of the geometric tolerances of the components on the kinematic motion deviations of the 5-axis machining centers. Main issues in the paper are summarized as follows.

(1) Modeling of geometric deviations of the 5-axis machining centers.

(2) Investigation of influences of geometric tolerances of guide-ways to table motions.

(3) Investigation of influences of table motions to kinematic motions of machine tools.

\section{Geometric Tolerances and Deviations of Features (Satonaka, et al., 2007)}

The geometric tolerances of the features specify the allowable areas named "tolerance zones," which constrain the position and orientation deviations of the associated features against the nominal features, as shown in Figure 1. The associated features and the nominal features mean the features of the manufactured products and the ideal features defined in the design phase, respectively. The geometric deviations of the associated features from the nominal features are represented by sets of parameters named "deviation parameters." For example, one position parameter $w$ and two rotational parameters $\alpha$ and $\beta$ are required to represent the geometric deviations of the associated plane features against the nominal plane features, for the case where the tolerance zone is given by the area between a pair of parallel planes.

In the research, the followings are assumed for the ease of the modeling and the analysis of the geometric deviations.

(1) The deviation parameters $\delta_{i}$ representing the position and orientation deviations of the associated features follow the normal distribution $N\left(\mu_{i}, \sigma_{i}\right)$, and $\mu_{i}=0$. Where, $\mu_{i}$ and $\sigma_{i}$ are the mean values and the standard deviations, respectively.

(2) The manufacturing processes of the components are well controlled, and the proportion of the non-conforming components is as small as a value $P d$ called "percent defective." Here, the non-conforming components mean the components, in which the toleranced features exceed the tolerance zones.

(3) Equation (1) represents the relationships between the standard deviations $\sigma_{i}$ of the deviation parameters of the toleranced features and the sizes of the tolerance zones.

$$
\sigma_{i}=\delta_{\text {imax }} / C_{p d}
$$

where,

$i$ : Indices of deviation parameters of the tolerance feature.

$\delta_{\text {imax }}$ : Tolerance limits of the deviation parameters $\delta_{i}$, if the other deviation parameters $\delta_{j}=0,(i \neq j)$.

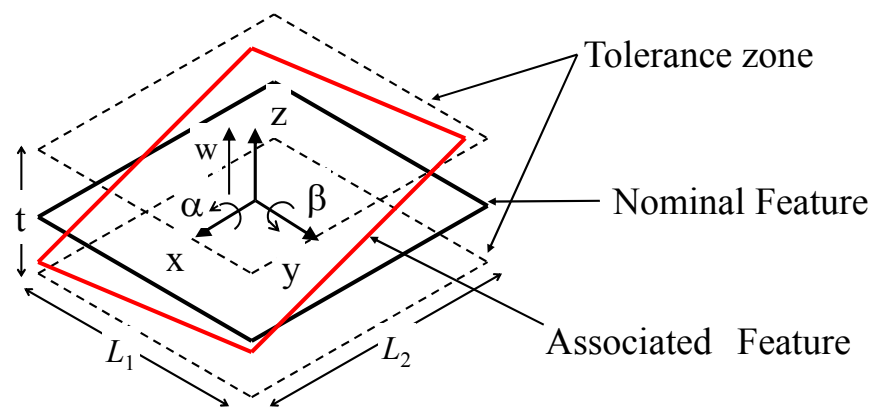

Fig. 1 Definition of geometric tolerance of plane faces 
Takahashi, Yoshida, Thasana, Sugimura, Iwamura and Tanimizu,

$C_{p d}$ : A constant representing the ratio of the maximum values $\delta_{\text {imax }}$ and the standard deviations $\sigma_{i}$. It is assumed that $C_{p d}$ has same value for all the deviation parameters of one tolerance feature.

Let us consider a case shown in Figure 1, as an example. The tolerance limit $\delta_{\text {imax }}$ are given as follows, under the conditons that $t$ is very smaller than $L_{1}$ and $L_{2}$.

$$
\begin{aligned}
& \delta_{1}=w, \delta_{2}=\alpha, \delta_{3}=\beta \\
& \delta_{1 \max }=t / 2, \delta_{2 \max }=t / L_{1}, \delta_{3 \max }=t / L_{2}
\end{aligned}
$$

where,

$L_{1}, L_{2}$ : Length and width of the plane feature.

$t$ : Tolerance values, e.g. the distance between two planes representing the tolerance zones.

The following inequality gives the conditions that the plane features are included within the tolerance zone between a pair of planes.

$$
-t / 2<\delta_{1}+L_{1} \delta_{2} / 2+L_{2} \delta_{3} / 2<t / 2
$$

The probability that the toleranced features are included within the tolerance zones is given by the following equation.

$$
1-P_{d}=\left(\frac{2}{\sqrt{2 \pi}}\right)^{3} \int_{0}^{C_{p d}} \int_{0}^{C_{p d}-x_{1}} \int_{0}^{C_{p d}-x_{1}-x_{2}}\left(\prod_{i=1}^{3} \exp \left(-\frac{x_{i}{ }^{2}}{2}\right)\right) \mathrm{d} x_{3} \mathrm{~d} x_{2} \mathrm{~d} x_{1}
$$

where,

$$
x_{1}=2 C_{p d} \delta_{1} / t, x_{2}=L_{1} C_{p d} \delta_{2} / t, x_{3}=L_{2} C_{p d} \delta_{3} / t
$$

If the percent defective $P d$ is less than $0.27 \%$, the constant $C_{p d}$ can be estimated as " $C_{p d}=5.83$ " for the cases of the plane features. As regards the cylinder features, " $C_{p d}=5.06$." The $C_{p d}$ values for the plane and the cylinder features are applied in the following analysis, in order to estimate the standard deviations of the deviation parameters of the guide-ways, based on the tolerance values.

\section{Kinematic Motions and Deviations of Rotary Tables and Linear Tables}

Figure 2 shows a typical example of rotary tables with vertical axis utilized for the machine tools. A base supports and guides a rotary table by a plane face and a cylindrical face called guide-ways, which are indicated by $a$ and $b$. In the case, the relative position and orientation of the table against the base are given by the following equation, if the guide-ways coincide with the nominal features.

$$
\mathbf{x}_{i}=\mathbf{A}_{a} \mathbf{A}_{a},{ }^{-1} \mathbf{x}_{i+1}=\mathbf{A}_{b} \mathbf{A}_{b},{ }^{-1} \mathbf{x}_{i+1}
$$

where,

$i$ : Indices representing the connecting sequences among the moving components of the machine tools.

$\mathbf{x}_{i}$ : Position vector of a point $\mathbf{P}$ in the base coordinate system.

$\mathbf{X}_{i+1}$ : Position vector of $\mathbf{P}$ in the table coordinate system.

$\mathbf{A}_{j}(j=a, b): 4 \times 4$ homogeneous transformation matrices representing the positions and orientations of the guide-ways $j$ in the base coordinate system.

$\mathbf{A}_{j},(j=a, b): 4 \times 4$ homogeneous transformation matrices representing the positions and orientations of the guide-ways $j$ in the table coordinate system.

However, the relative positions of the table against the base are influenced by the position and orientation deviations of the guide-ways, if the guide-ways do not coincide with the nominal features. The positions of the table against the base are represented by the following equation corresponding to the individual guide-ways.

$$
\mathbf{X}_{i}=\mathbf{A}_{j} \mathbf{D}_{j} \mathbf{D}_{j},{ }^{-1} \mathbf{A}_{j}{ }^{-1} \mathbf{X}_{i+1}(j=a, b)
$$




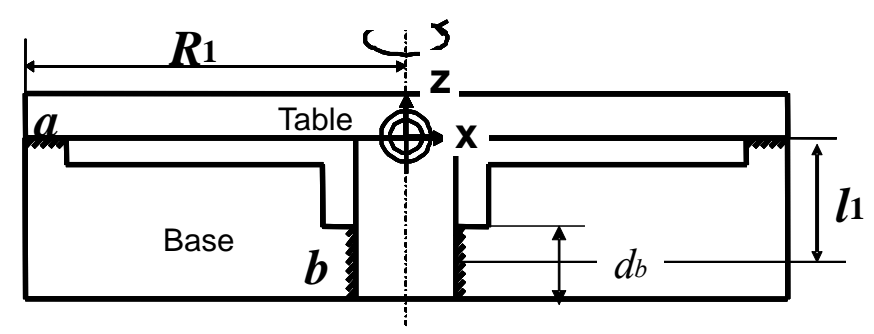

Fig. 2 Vertical rotary tables (C-axis table) and their guide-ways

where,

$\mathbf{D}_{j}, \mathbf{D}_{j}: 4 \times 4$ homogeneous transformation matrices representing the position and orientation deviations of the pair of features constituting the guide-ways $j$.

Equation (6) means that various values of the relative position of the table against the base are obtained for the individual guide-ways. A systematic method was proposed by considering the priority among the guide-ways to determine the relative positions of the tables against the base. The followings summarize the models of the rotary tables and the linear tables (Satonaka, et al., 2008; Sugimura, et al.,2012).

\section{(1) Vertical Rotary Tables}

The kinematic motion deviations of the vertical rotary tables shown in Figure 2 are formulated in Eq. (7), under the assumption that the priority among the guide-ways is " $a>b$." Where, " $a>b$ " means that the higher priority is given to the guide-way $a$ against $b$ to determine the positions and orientations of the tables to the bases considering the geometric deviations of the guide-ways.

$$
\begin{aligned}
& \mathbf{A}_{6}(\theta)=\left(\begin{array}{cccc}
\cos \theta & -\sin \theta & \delta_{V \beta 1} & \delta_{V x} \\
\sin \theta & \cos \theta & \delta_{V \alpha 1} & \delta_{V y} \\
\delta_{V \beta 2} & \delta_{V \alpha 2} & 1 & \delta_{V z} \\
0 & 0 & 0 & 1
\end{array}\right) \\
& \delta_{V \alpha 1}=-\beta_{b 2} \sin \theta+\alpha_{b 2} \cos \theta-\alpha_{b 1} \\
& \delta_{V \alpha 2}=\beta_{b 1} \sin \theta+\alpha_{b 1} \cos \theta-\alpha_{b 2} \\
& \delta_{V \beta 1}=-\beta_{b 2} \cos \theta-\alpha_{b 2} \sin \theta+\beta_{b 1} \\
& \delta_{V \beta 2}=-\beta_{b 1} \cos \theta+\alpha_{b 1} \sin \theta+\beta_{b 2} \\
& \delta_{V x}=-l_{1} \beta_{b 2} \cos \theta-l_{1} \alpha_{b 2} \sin \theta+l_{1} \beta_{b 1}-\delta_{x b 2} \cos \theta+\delta_{y_{b 2}} \sin \theta+\delta_{x b 1} \\
& \delta_{V y}=-l_{1} \beta_{b 2} \sin \theta+l_{1} \alpha_{b 2} \cos \theta-l_{1} \alpha_{b 1}-\delta_{x b 2} \sin \theta-\delta_{y_{b 2}} \cos \theta+\delta_{y_{b 1}} \\
& \delta_{V z}=\delta_{z a 1}-\delta_{z a 2}
\end{aligned}
$$

where,

$\mathbf{A}_{j}(j=1,2, \cdots, 6)$ : Motions of linear tables in $\mathrm{x}-, \mathrm{y}$, and $\mathrm{z}$-directions and ones of rotary tables around $\mathrm{x}-, \mathrm{y}$, and $\mathrm{z}$-axes. The details will be shown in Eq. (10).

$\theta$ : Rotation angle of table.

$\alpha_{i j}, \beta_{i j}, \gamma_{i j}$, : Orientation deviations the $j$-th geometric feature of guide-way $i$ around $\mathrm{x}, \mathrm{y}$ and $\mathrm{z}$ axis of the rotary tables. $\delta_{x i j}, \delta_{y i j}, \delta_{z i j}$ : Position deviations the $j$-th geometric feature of guide-way $i$ along $\mathrm{x}, \mathrm{y}$ and $\mathrm{z}$ axis of the rotary tables.

\section{(2) Horizontal Rotary tables}

For the cases of the horizontal rotary tables shown in Figure 3, the kinematic motion deviations are formulated in Eq. (8), under the assumption that the priority among the guide-ways is " $(c=e)>(d=f)$." Where, " $(c=e)$ " means that there is not any priority between the guide-ways $\boldsymbol{c}$ and $\boldsymbol{e}$ to determine the positions and orientations of the tables to 
Takahashi, Yoshida, Thasana, Sugimura, Iwamura and Tanimizu,

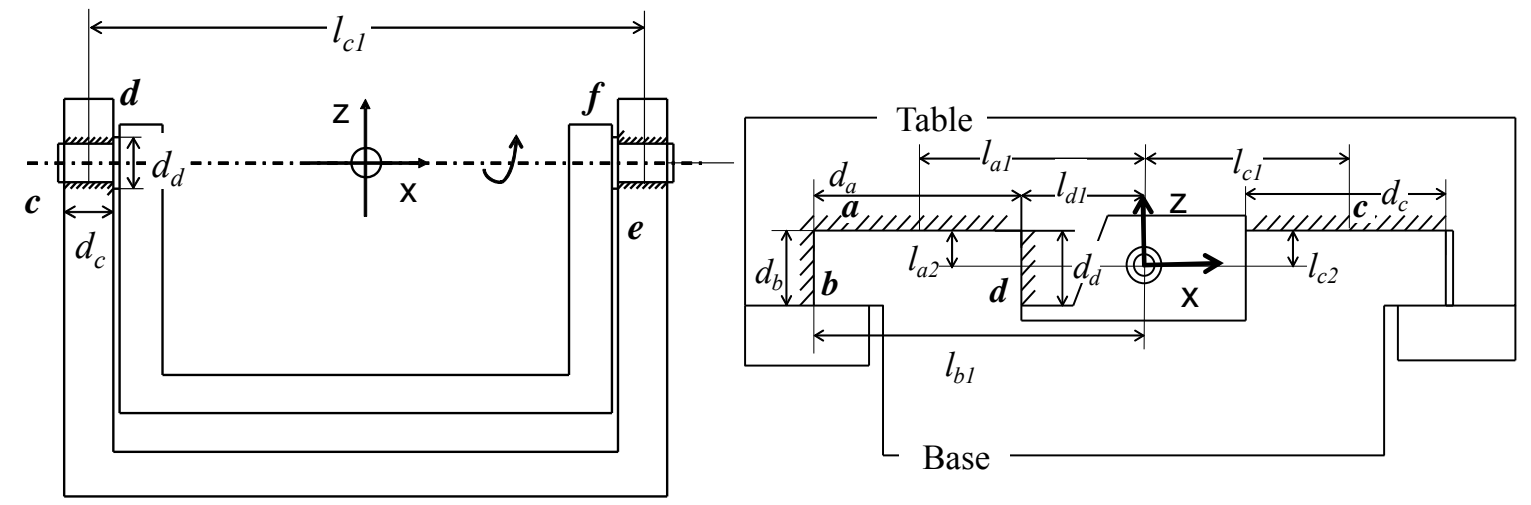

Fig. 3 Horizontal Rotary tables (A-axis table)

Fig. 4 Liner motion tables

the bases considering the geometric deviations of the guide-ways.

$$
\begin{aligned}
& \mathbf{A}_{4}(\varphi)=\left(\begin{array}{cccc}
1 & \frac{1}{2} \delta_{H \gamma 1} & \frac{1}{2} \delta_{H \beta 1} & \frac{1}{2} \delta_{H x} \\
\frac{1}{2} \delta_{H \gamma 2} & \cos \varphi & -\sin \varphi & \frac{1}{2} \delta_{H y} \\
\frac{1}{2} \delta_{H \beta 2} & \sin \varphi & \cos \varphi & \frac{1}{2} \delta_{H z} \\
0 & 0 & 0 & 1
\end{array}\right) \\
& \delta_{H \beta 1}=-\beta_{c 2}-\beta_{e 2}+\left(\gamma_{c 1}+\gamma_{e 1}\right) \sin \varphi+\left(\beta_{c 1}+\beta_{e 1}\right) \cos \varphi \\
& \delta_{H \beta 2}=-\beta_{c 1}-\beta_{e 1}-\left(\gamma_{c 2}+\gamma_{e 2}\right) \sin \varphi+\left(\beta_{c 2}+\beta_{e 2}\right) \cos \varphi \\
& \delta_{H \gamma 1}=\gamma_{c 2}+\gamma_{e 2}-\left(\gamma_{c 1}+\gamma_{e 1}\right) \cos \varphi+\left(\beta_{c 1}+\beta_{e 1}\right) \sin \varphi \\
& \delta_{H \gamma 2}=\gamma_{c 1}+\gamma_{e 1}-\left(\gamma_{c 2}+\gamma_{e 2}\right) \cos \varphi-\left(\beta_{c 2}+\beta_{e 2}\right) \sin \varphi \\
& \delta_{H x}=\delta_{x d 1}-\delta_{x d 2}+\delta_{x f 1}-\delta_{x f 2} \\
& \left.\delta_{H y}=\delta_{y c 1}+\delta_{y e 1}-\left(\delta_{y c 2}+\delta_{y e 2}\right) \cos \varphi+\left(\delta_{z c 2}+\delta_{z e 2}\right) \sin \varphi-\frac{l_{c 1}}{2}\left\{\left(\gamma_{e 1}-\gamma_{c 1}\right)-\left(\gamma_{e 2}-\gamma_{c 2}\right) \cos \varphi-\left(\beta_{e 2}-\beta_{c 2}\right) \sin \varphi\right)\right\} \\
& \left.\delta_{H z}=\delta_{z c 1}+\delta_{z e 1}-\left(\delta_{z c 2}+\delta_{z e 2}\right) \cos \varphi-\left(\delta_{y c 2}+\delta_{y e 2}\right) \sin \varphi+\frac{l_{c 1}}{2}\left\{\left(\beta_{e 1}-\beta_{c 1}\right)-\left(\beta_{e 2}-\beta_{c 2}\right) \cos \varphi+\left(\gamma_{e 2}-\gamma_{c 2}\right) \sin \varphi\right)\right\}
\end{aligned}
$$

where,

$\varphi$ : Rotational angle of the table.

(3) Linear Tables

The kinematic motion deviations of $y$-axis liner tables shown in Figure 4 are formulated in the following equations, under the assumption that the priority among the guide-ways is " $(a=c)>(b=d)$."

$$
\mathbf{A}_{2}(y)=\left(\begin{array}{cccc}
1 & -\frac{\delta_{\gamma b}+\delta_{\gamma d}}{2} & \frac{\delta_{\beta a}+\delta_{\beta c}}{2} & \frac{1}{2}\left\{\left(\delta_{u b}+\delta_{u d}\right)-\left(\delta_{\gamma b}^{i}+\delta_{\gamma d}^{i}\right) y\right\} \\
\frac{\delta_{\gamma}+\delta_{\gamma d}}{2} & 1 & -\frac{\delta_{\alpha a}+\delta_{\alpha c}}{2} & y \\
-\frac{\delta_{\beta a}+\delta_{\beta c}}{2} & \frac{\delta_{\alpha a}+\delta_{\alpha c}}{2} & 1 & \frac{1}{2}\left\{\left(\delta_{w a}+\delta_{w c}\right)+\frac{1}{2}\left(l_{a 1}+l_{c 1}\right)\left(\delta_{\beta a}+\delta_{\beta c}\right)+\left(\delta_{\alpha a}^{i}+\delta_{\alpha c}^{i}\right) y\right\} \\
0 & 0 & 0 & 1
\end{array}\right)
$$

where,

$y$ : y-direction travel.

$\delta_{p q}$ : Differences of the position and orientation deviations of the features in base side and table side consisting of the $q$-th guide-ways of in the $p$-th directions.

$p(=\alpha, \beta, \gamma, u, v, w)$ : Components of the position and orientation deviations. 
Takahashi, Yoshida, Thasana, Sugimura, Iwamura and Tanimizu,

$q(=a, b, c, d):$ Guide-ways.

$\delta_{\alpha a}^{i}$ : Position and orientation deviations of the features in base side of the $q$-th guide-ways of in the $p$-th directions.

\section{Analysis of Kinematic Motion Deviations of Five-Axis Machining Centers 4.1 Modeling of Kinematic Motions}

The five-axis machining centers are composed of two rotary tables and three liner tables shown in Figures 2, 3 and 4. Three types of five-axis machining centers shown in Figure 5 are considered here for the analysis. The shape generation motions of the machining centers are summarized in Eq. (10)

Type 1: $\mathbf{r}_{w}=\mathbf{A}_{3}\left(d_{1}\right) \mathbf{A}_{6}(\theta) \mathbf{A}_{3}\left(d_{2}\right) \mathbf{A}_{4}(\varphi) \mathbf{A}_{3}\left(d_{3}\right) \mathbf{A}_{3}(x) \mathbf{A}_{3}\left(d_{4}\right) \mathbf{A}_{2}(y) \mathbf{A}_{3}\left(d_{5}\right) \mathbf{A}_{3}(z) \mathbf{r}_{t}$

Type 2: $\mathbf{r}_{w}=\mathbf{A}_{3}\left(d_{1}\right) \mathbf{A}_{6}(\theta) \mathbf{A}_{3}\left(d_{2}\right) \mathbf{A}_{1}(x) \mathbf{A}_{3}\left(d_{3}\right) \mathbf{A}_{2}(y) \mathbf{A}_{3}\left(d_{4}\right) \mathbf{A}_{3}(z) \mathbf{A}_{3}\left(d_{5}\right) \mathbf{A}_{4}(\varphi) \mathbf{r}_{t}$

Type 3: $\mathbf{r}_{w}=\mathbf{A}_{3}\left(d_{1}\right) \mathbf{A}_{1}(x) \mathbf{A}_{3}\left(d_{2}\right) \mathbf{A}_{2}(y) \mathbf{A}_{3}\left(d_{3}\right) \mathbf{A}_{3}(z) \mathbf{A}_{3}\left(d_{4}\right) \mathbf{A}_{6}(\theta) \mathbf{A}_{3}\left(d_{5}\right) \mathbf{A}_{4}(\varphi) \mathbf{r}_{t}$

where,

$\mathbf{A}_{1}(x): \mathrm{x}$-axis linear motion

$\mathbf{A}_{2}(y): \mathrm{y}$-axis liner motion

$\mathbf{A}_{3}(z):$ z-axis liner motion

$\mathbf{A}_{4}(\varphi):$ A-axis rotary motion

$\mathbf{A}_{6}(\theta)$ : C-axis rotary motion

$\mathbf{A}_{3}\left(d_{i}\right)$ : Translation between the tables

$d_{i}$ : Distances in z-direction between pairs of constituting tables. They are summarized in Table 1.

\subsection{Analysis of Kinematic Motion Deviations of Five-Axis Machining Centers}

The dimensions and the strokes shown in Table 2 are given to $\mathrm{x}-, \mathrm{y}$ - and $\mathrm{z}$-tables for three types of machining centers. The dimensions are same for all types of machining centers. Table 3 summarizes the dimensions of the rotary tables, which are designed for the individual types of machining centers.

The kinematic motion deviations of the tools against the workpieces are analyzed by applying Eq. (13), according to the following procedure.

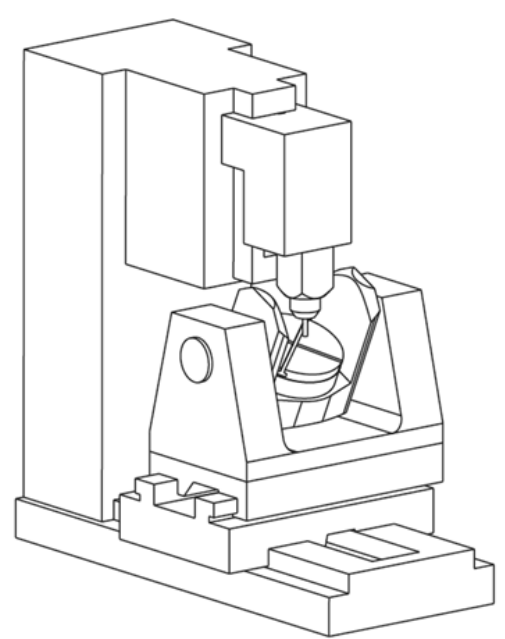

(a) Type 1:Workpiece rotation type

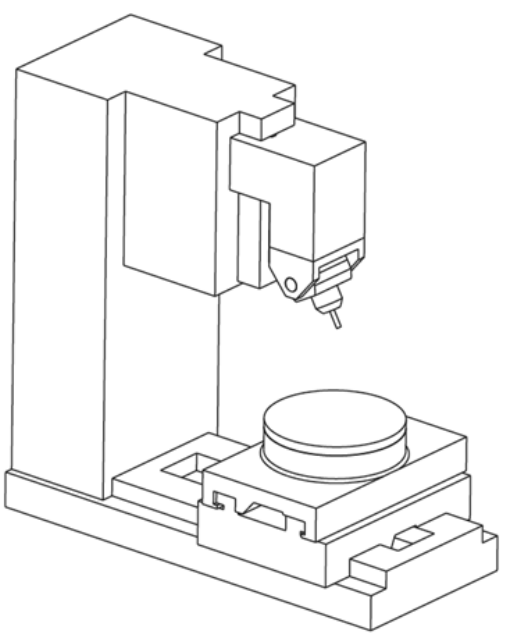

(b) Type 2:Tool \& workpiece rotation type

Fig. 5 Five-axis machining centers

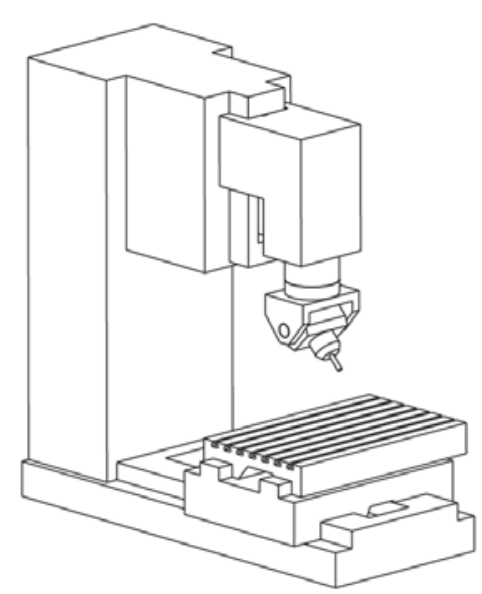

(c ) Type 3:Tool rotation type 
Takahashi, Yoshida, Thasana, Sugimura, Iwamura and Tanimizu,

Journal of Advanced Mechanical Design, Systems, and Manufact, Vol.8, No.4 (2014)

STEP 1: Set the table positions

The table positions are set as shown in Table 4 to analyze the kinematic motion deviations for the individual positions.

STEP 2: Set the initial tolerance values for all the guide-ways

All the tolerance values are set that $t=50 \mu \mathrm{m}$ for all the guide-ways shown in Figures 2, 3 and 4 . These values are assumed as the initial values. The standard deviations of the position and orientation deviations of the tools against the workpieces are estimated by applying Eq. (13), based on the formulas given by Equations (7), (8) and (9) representing the kinematic motions and their standard deviations of the rotary and the linear tables. The standard deviations of these table motions are also estimated, based on the standard deviations of the position and orientation deviations of the individual guide-ways given in Eq. (1).

STEP 3 :Set the small tolerance value for each guide-way

A tolerance values of one of the guide-ways are set that $t=5 \mu \mathrm{m}$, which is $1 / 10$ of the initial values, in order to clarify the effects of the improvement of the tolerance values of individual guide-ways. The standard deviations of the position and orientation deviations of the tools against the workpieces are estimated in same manner shown in (2), and the obtained standard deviations are compared by the following equation to clarify the effects of the individual guide-ways.

$$
i r=\frac{S t_{50}-S t_{5}}{S t_{50}} \times 100
$$

where,

$S t_{50}$ : Standard deviations obtained based on initial tolerance values.

$S t_{5}$ : Standard deviations obtained based on improved tolerance values.

Table 1 Distances between a pair of connecting tables

\begin{tabular}{|c|c|c|c|}
\hline Sizes $(\mathrm{mm})$ & Type1 & Type2 & Type3 \\
\hline$d_{1}$ & -20 & -20 & -30 \\
\hline$d_{2}$ & 60 & -90 & -70 \\
\hline$d_{3}$ & -230 & -70 & 390 \\
\hline$d_{4}$ & -70 & 400 & -70 \\
\hline$d_{5}$ & 450 & -30 & -30 \\
\hline
\end{tabular}

Table 2 Sizes and strokes of linear tables for types 1, 2 and 3.

\begin{tabular}{|c|c|c|c|}
\hline Sizes $(\mathrm{mm})$ & $\mathrm{x}$-table & $\mathrm{y}$-table & z-table \\
\hline$l_{a 1}$ & 95 & 110 & 40 \\
\hline$l_{a 2}$ & 12 & 20 & 30 \\
\hline$l_{b 1}$ & 125 & 160 & 55 \\
\hline$l_{c 1}$ & 95 & 110 & 40 \\
\hline$l_{c 2}$ & 12 & 20 & 30 \\
\hline$l_{d 1}$ & 65 & 60 & 25 \\
\hline$d_{a}$ & 60 & 100 & 30 \\
\hline$d_{b}$ & 30 & 40 & 60 \\
\hline$d_{c}$ & 60 & 100 & 30 \\
\hline$d_{d}$ & 30 & 40 & 60 \\
\hline length $\mathrm{mm}$ & 500 & 350 & 200 \\
\hline stroke $\mathrm{mm}$ & $-200 \leqq x \leqq 200$ & $-175 \leqq y \leqq 175$ & $0 \leqq z \leqq 190$ \\
\hline
\end{tabular}


Takahashi, Yoshida, Thasana, Sugimura, Iwamura and Tanimizu, Journal of Advanced Mechanical Design, Systems, and Manufact, Vol.8, No.4 (2014)

Table 3 Sizes of rotary tables for types 1, 2 and 3.

\begin{tabular}{|c|c|c|c|c|}
\hline \multirow{2}{*}{ Tables } & \multirow{2}{*}{ Sizes $(\mathrm{mm})$} & \multicolumn{3}{|c|}{ Types } \\
\cline { 3 - 5 } & & Type1 & Type2 & Type3 \\
\hline \multirow{3}{*}{ C-axis table } & $d_{b}$ & 30 & 30 & 20 \\
\cline { 2 - 5 } & $l_{1}$ & 45 & 45 & 22.5 \\
\cline { 2 - 5 } & $R_{1}$ & 102.5 & 102.5 & 54 \\
\hline \multirow{3}{*}{ A-axis table } & $d_{c}\left(=d_{e}\right)$ & 25 & 25 & 12.5 \\
\cline { 2 - 5 } & $d_{d}\left(=d_{f}\right)$ & $\phi 30$ & $\phi 30$ & $\phi 15$ \\
\cline { 2 - 5 } & $l_{c 1}$ & 340 & 120 & 120 \\
\hline
\end{tabular}

Table 4 Table positions to be set in analysis

\begin{tabular}{|c|c|c|c|c|c|}
\hline Positions & $\begin{array}{c}\text { X-axis } \\
x(\mathrm{~mm})\end{array}$ & $\begin{array}{c}\mathrm{y} \text {-axis } \\
(\mathrm{mm})\end{array}$ & $\begin{array}{c}\text { z-axis } \\
z(\mathrm{~mm})\end{array}$ & $\begin{array}{c}\text { A-axis } \\
\varphi \text { (degree) }\end{array}$ & $\begin{array}{c}\text { C-axis } \\
\theta \text { (degree) }\end{array}$ \\
\hline Positoin-1 & 200 & 175 & 190 & 90 & 180 \\
\hline Position-2 & 100 & 90 & 95 & 45 & 90 \\
\hline Position-3 & 0 & 0 & 0 & 0 & 0 \\
\hline
\end{tabular}

The ir values obtained here show the improvement ratio of the tolerance values of the individual guide-ways. When the ir value of one guide-way is high, the improvement of the tolerance values of the guide-way is very effective to improve the whole kinematic deviations of the five-axis machining centers.

Figures 6,7 , and 8 summarize the analysis results of the ir values for all the table positions and all the types of the machining centers.

It is found that the improvement ratio of the A-axis and $\mathrm{C}$-axis rotary tables are higher than ones of the linear tables for all cases of the analysis conditions. In particular, the guide-ways $\boldsymbol{c}$ and $\boldsymbol{e}$ of A-axis and the guide-way $\boldsymbol{a}$ of $\mathrm{C}$-axis are larger effects than the other guide-ways, since these guide-ways have higher priority to determine the kinematic motion deviations for the rotary tables, as shown in Eqs. (7) and (8).

As regards the types of machining centers, the type 3 has different characteristics from the others. The improvement ratio of the $\mathrm{x}$-axis and $\mathrm{y}$-axis linear tables are relatively high against the other types of machining centers. The type 3 machining centers have no rotational axes in the workpiece side, and the effects of the rotational motion deviations in tool side may be reduced, since they have smaller arm lengths between the rotational center of the tool side and the cutting points of the tools.

It is understood that the proposed model is effective to analyze the effects of the tolerance values of the guide-ways to the kinematic motion deviations of the tools against the workpieces in the five-axis machining centers.

\section{Conclusions}

A mathematical model has been proposed to represent the kinematic motion deviations of the five-axis machining centers. Following remarks are concluded.

(1) A set to models are proposed to estimate the kinematic motion deviations of the rotary tables and the linear tables, based on the tolerance values of the guide-ways of the tables. The models give the mathematical relationships between the kinematic deviations of tables and the geometric tolerances of the guide-ways. 
Takahashi, Yoshida, Thasana, Sugimura, Iwamura and Tanimizu, Journal of Advanced Mechanical Design, Systems, and Manufact, Vol.8, No.4 (2014)

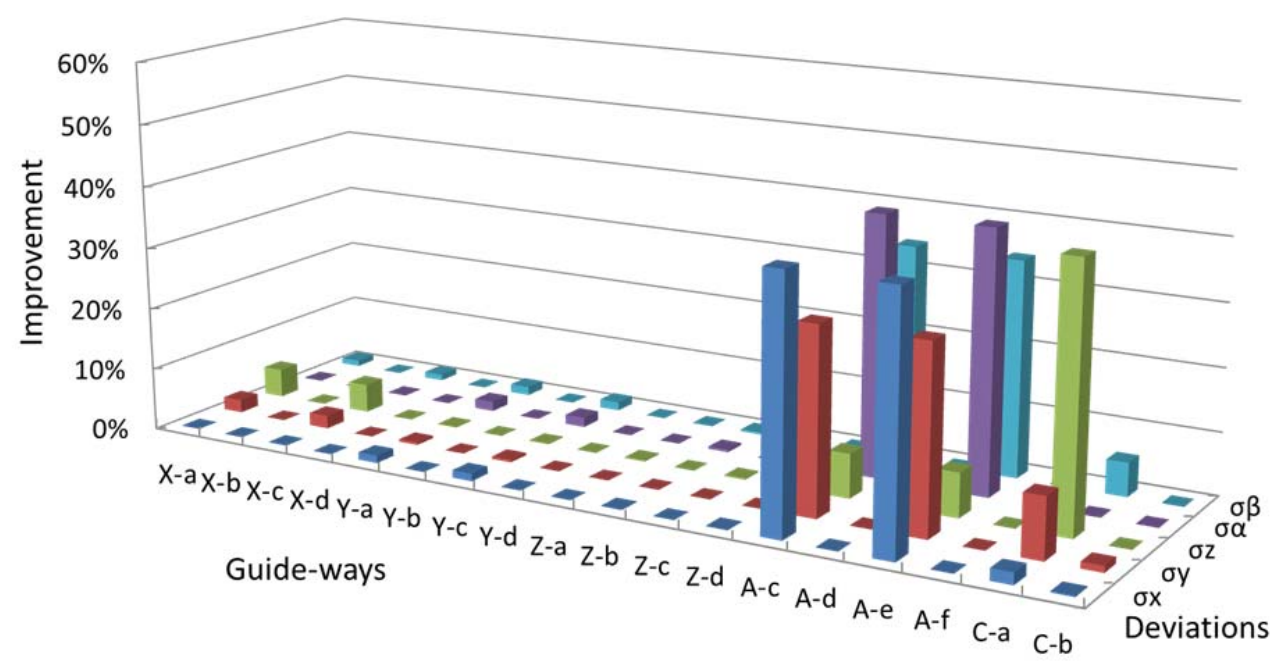

(a) Position-1

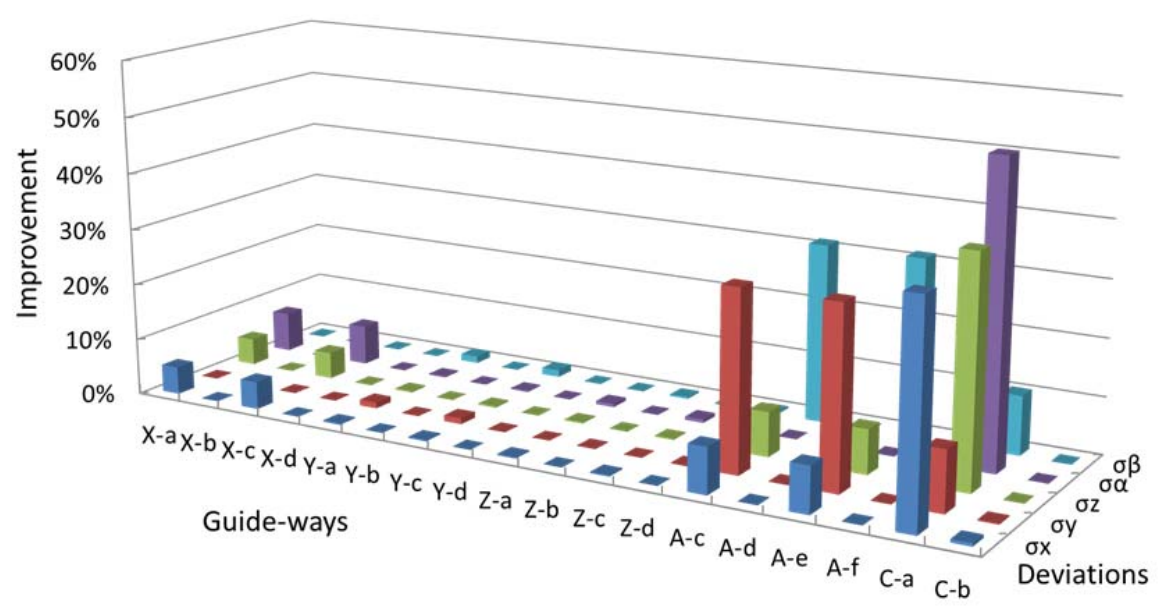

(b) Position-2

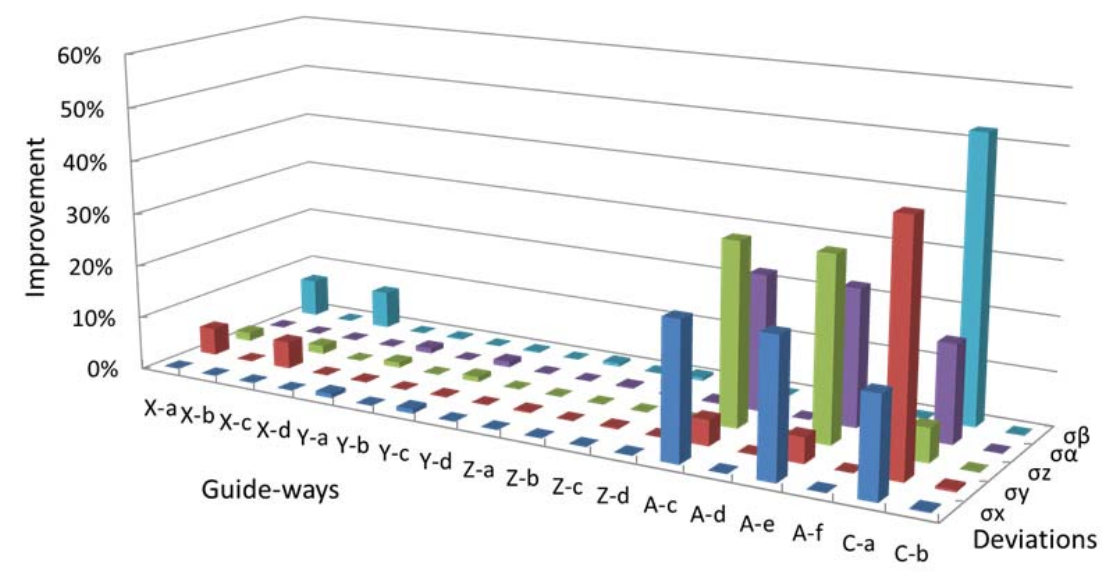

(c) Position-3

Fig. 6 Analysis results of type 1 machining center 
Takahashi, Yoshida, Thasana, Sugimura, Iwamura and Tanimizu, Journal of Advanced Mechanical Design, Systems, and Manufact, Vol.8, No.4 (2014)

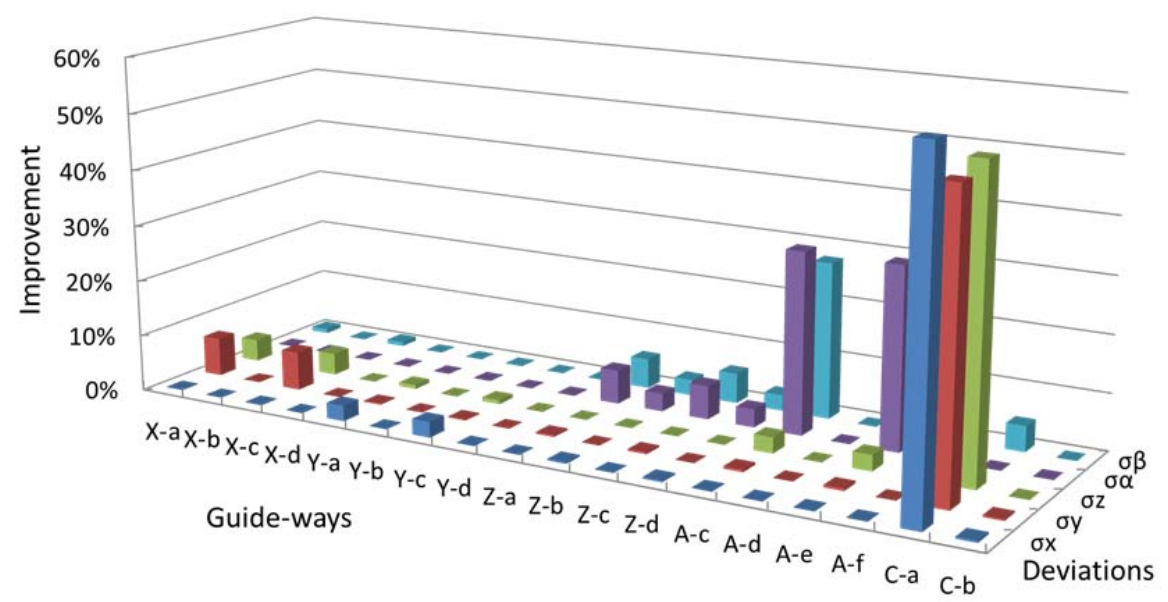

(a) Position-1

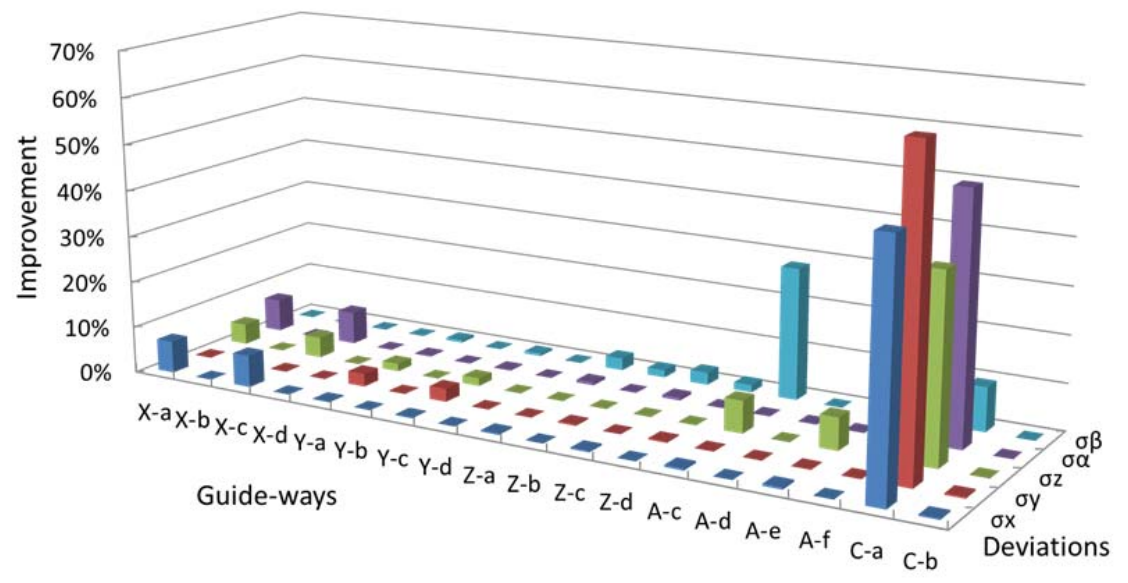

(b) Position-2

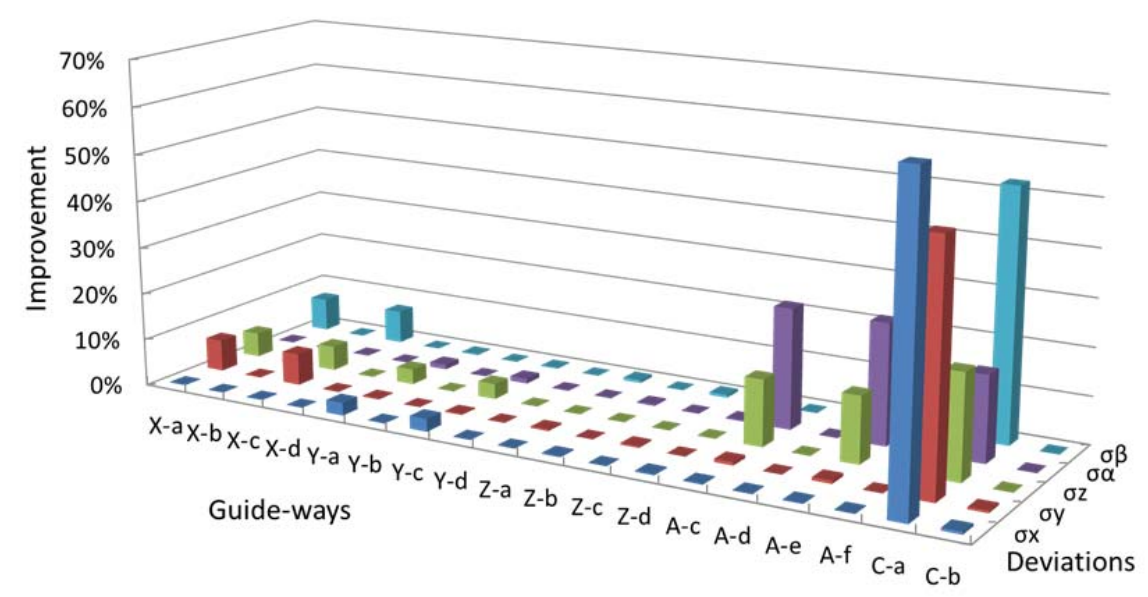

(c) Position-3

Fig. 7 Analysis results of type 2 machining center 
Takahashi, Yoshida, Thasana, Sugimura, Iwamura and Tanimizu, Journal of Advanced Mechanical Design, Systems, and Manufact, Vol.8, No.4 (2014)

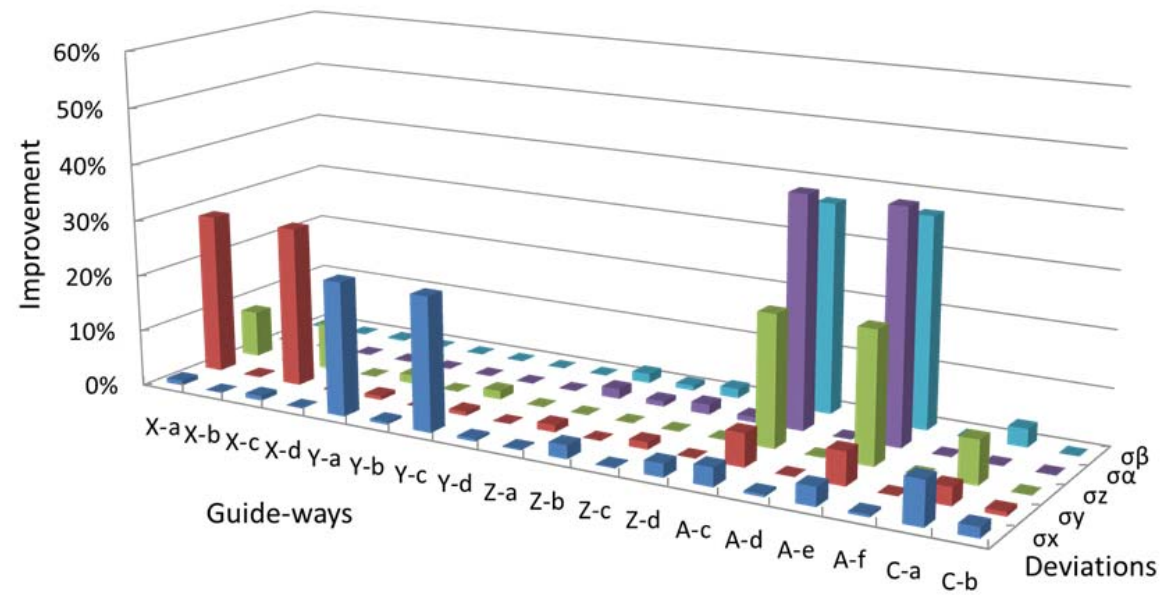

(a) Position-1

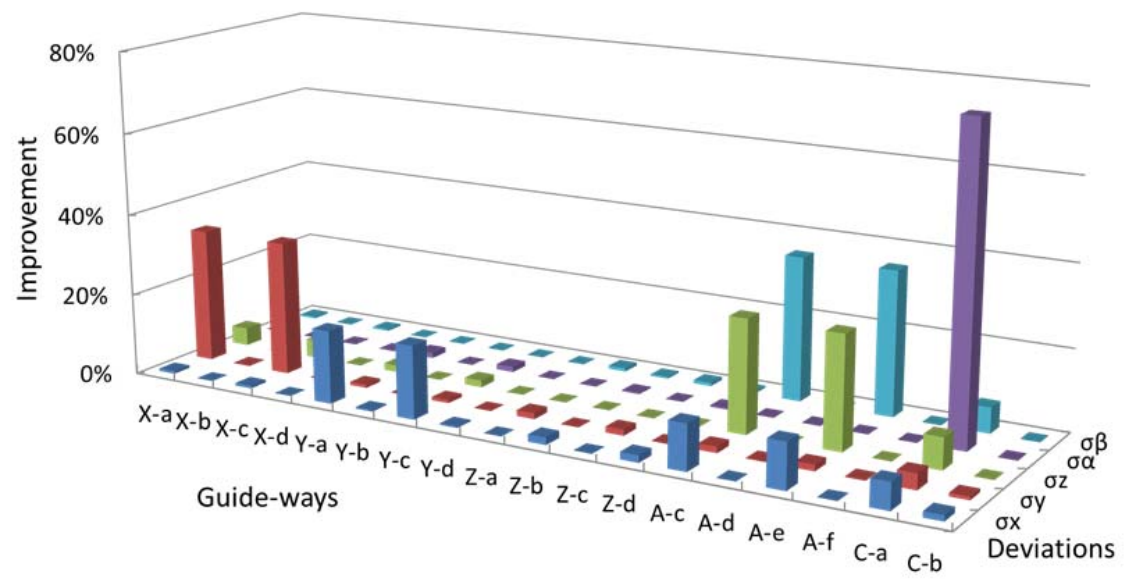

(b) Position-2

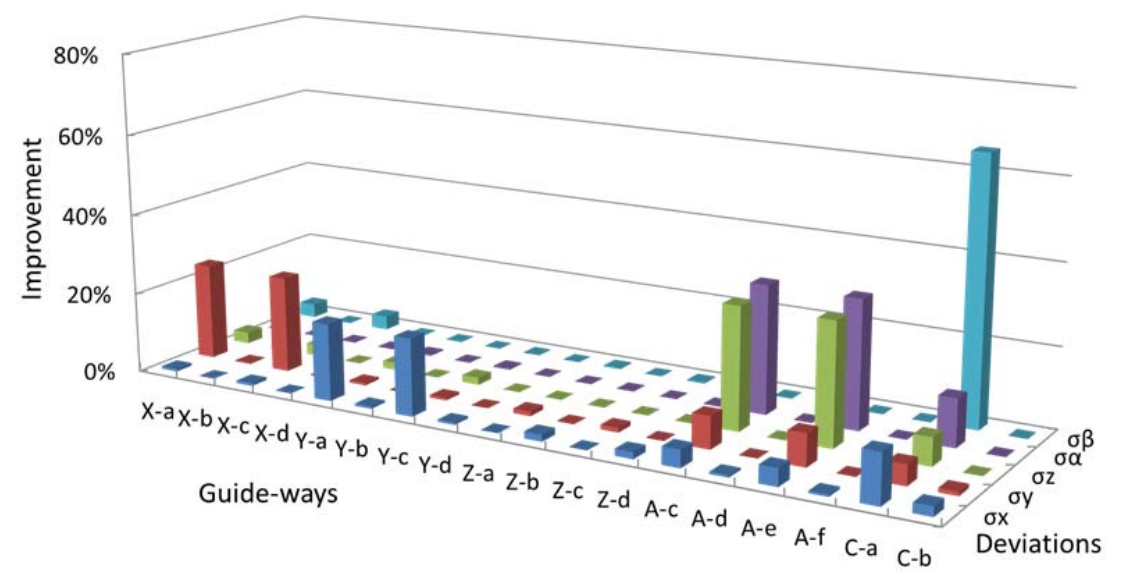

(c) Position-3

Fig. 8 Analysis results of type 3 machining center 
Takahashi, Yoshida, Thasana, Sugimura, Iwamura and Tanimizu,

Journal of Advanced Mechanical Design, Systems, and Manufact, Vol.8, No.4 (2014)

(2) A set of models are proposed to estimate the kinematic motion deviations for three types of the five-axis machining centers by combining the models of the rotary tables and the linear tables. The models proposed here provide us with systematic methods to estimate the standard deviations of the position and orientation deviations of the tools against the workpieces, based on tolerance values of the guide-ways

(3) The kinematic motion deviations of the five-axis machining centers are estimated by applying the proposed models. It is understood that the kinematic motion deviations are affected by the geometric tolerances of the guide-ways and that the geometric tolerances of the guide-ways of rotary tables have higher effects on the kinematic motion deviations than the ones of linear tables.

\section{References}

Ngoi, B.K.A., Lim, B.H. and Ang, P.S., Nexus method for stack analysis of geometric dimensioning and tolerancing (GDT) problems, International Journal of Production Research, Vol. 38, No. 1(2000), pp.21-37.

Nigam, S.D. and Turner, J.U., Review of statistical approaches to tolerance analysis, Computer-Aided Design, Vol. 27, No. 1(1995), pp.6-15.

Reshetov, D.N. and Portman, V.T., 1988, Accuracy of machine tools, APME Press.

Roy, R., Liu, C.R. and Woo, T.C., Review of dimensioning and tolerancing: representation and processing, Computer-Aided Design, Vol. 23, No. 3(1991), pp.466-483.

Sakamoto, S. and Inasaki, I., Error analysis of precision machine tools, Proceeding of the 3rd International Conference of Ultraprecision in Manufacturing Engineering(1994), pp.245-250.

Satonaka, N., Sugimura, N., Tanimizu, Y. and Iwamura, K., Analysis of kinematic motion deviations of machining centers based on geometric tolerances, Proceedings of the 4th LEM-21(2007), pp.629-634.

Satonaka, N., Sugimura, N., Tanimizu, Y. and Iwamura, K., A study on modeling and analysis of kinematic motion deviations of machine tools (2nd Report, Modeling and analysis of linear tables and machine tools), Transactions of the Japan Society of Mechanical Engineers, Series C, Vol. 74, No. 737(2008), pp.198-205(in Japanese).

Sugimura, N., Iwata, K., and Oba, F., Formation of shape generation processes of machine tools, Proceedings of IFAC' 81, Vol. XIV(1981), pp.158-163.

Sugimura, N. and Mitani, S., Analytical approach for design of alignment errors of machine tools, Proc. of 1998 Pacific Conference on Manufacturing(1998), pp.371-374.

Sugimura, N., Watabiki, H., Thasana, W., Iwamura, K. and Tanimizu, Y., Analysis of kinematic motion deviations of rotary tables based on geometric tolerances. Journal of Advanced Mechanical Design, Systems and Manufacturing, Vol. 6, No. 7 (2012), pp.1132-1142.

Voelcker, H.B., A current perspective on tolerancing and metrology, CRTD-Vol.27, International Forum on Dimensional Tolerancing and Metrology(1993), pp.46-68. 